\title{
Advanced Computer Drawing Envelopes of Transmittance Spectra of Thin Film Specimens
}

\author{
G. M. Gavrilov ${ }^{1}$, D. A. Minkov' ${ }^{1}$, E. Marquez ${ }^{2}$, S. M. F. Ruano ${ }^{3}$ \\ Department of Electronics, Computer Systems and Technologies, College of Energetics and Electronics, \\ Technical University-Sofia, Botevgrad, Bulgaria ${ }^{1}$ \\ Department de Fisica de la Materia Condensada, Facultad de Ciencias, Universidad de Cadiz, Cadiz, Spain ${ }^{2}$ \\ Energy Department, Photovoltaic Solar Energy Unit, CIEMAT, Madrid, Spain ${ }^{3}$
}

\begin{abstract}
Drawing accurate envelopes of a transmittance spectrum is the main factor limiting the accuracy of characterization of one thin film on transmitting substrate specimens, using the method of Swanepoel. An advanced algorithm is proposed for computer drawing accurate envelopes, over the entire spectrum, which takes into account the following issues not considered in the existing algorithms: the wider spectrum for UV/visible/NIR compared to UV/visible spectrophotometers, the absorption in the substrate, and the increasing demand for accuracy in the spectral region of strong absorption in the film. This algorithm uses only one-step smoothing the spectrum, and piecewise cubic Hermite polynomial interpolation which is shown to be superior to cubic spline interpolation for constructing envelopes of such spectra. Boundary points, additional points, and supplementary points are included in the interpolation. The performance of the algorithm is demonstrated for two model specimens. Envelopes are also computer drawn for experimental spectra of two specimens of a-Si film on glass substrates.
\end{abstract}

Keywords: thin film on glass specimen, transmittance spectrum, two envelopes of the spectrum, computer drawing.

\section{INTRODUCTION}

Optical characterization of thin films is required for numerous engineering applications [1-4]. A review of the methods for characterization of thin dielectric, semiconductor, and insulator films, using light transmittance measurements [5], indicates that one of the most popular methods is the one proposed by Swanepoel in 1983 [6]. In this method, the film is positioned on an almost transparent substrate, and one transmittance spectrum $T(\lambda)$ of this specimen is needed, where $T$ is the transmittance, and $\lambda$ is the wavelength of light. This spectrum should exhibit an interference pattern, including several maxima and minima, which is a result of the light interference from the top and bottom surfaces of the film [6-7]. The method uses two smooth envelopes drawn around the maxima and the minima of the transmittance spectrum. These envelopes have only several common tangential points with $\mathrm{T}(\lambda)$, and do not cross it. The upper envelope $T_{+}(\lambda)$ lies above $T(\lambda)$, i.e. $T_{+}(\lambda) \geq T(\lambda)$, and the lower envelope $T_{-}(\lambda)$ lies below $T(\lambda)$, i.e. $T_{-}(\lambda) \leq T(\lambda)$.

The advantages of the method of Swanepoel are a combination of: absence of any model for the refractive index dispersion $\mathrm{n}(\lambda)$ or the spectrum; and high accuracy due to using additional information from both envelopes, and from the positions of the interference fringes of the spectrum [5-6].

Drawing accurate envelopes is recognized to be the main factor limiting the accuracy of optical characterizations using the method of Swanepoel - see [6] and [8]. The first algorithm for computer drawing envelopes of spectra, exhibiting interference pattern, has been proposed by McClain et al. in 1991 [9]. It is based mathematically on B-spline interpolation, and multi-step iteration of splines for determining the tangential points. In [9], the envelopes are drawn only along tangential points positioned close to apparent extrema of $T(\lambda)$. Such tangential points though occur only in the spectral region of negligible to medium absorption in the film [6], which limits the spectral region of applicability of the algorithm. Nevertheless, this algorithm is often-used for drawing envelopes of transmittance spectra, in optical characterization by the method of Swanepoel [10-11].

Furthermore, in [9] are shown computed data for $n(\lambda=$ $300 \div 700 \mathrm{~nm})$ of a film $\left(\mathrm{Y}_{2} \mathrm{O}_{3}\right)_{0.9}:\left(\mathrm{SiO}_{2}\right)_{0.1}$ of the rare earth element Ittrium. It is also known that a very large number of materials of thin films satisfy the single-oscillator model, whereat the dependence of $\left(n^{2}-1\right)^{-1}$ versus $\left(\lambda^{-2}\right)$ is approximated by a straight line, representing a plot for the Wemple-DiDomenico model [12-14]. In this regard, we made such a plot, using the data for the $\left(\mathrm{Y}_{2} \mathrm{O}_{3}\right)_{0.9}:\left(\mathrm{SiO}_{2}\right)_{0.1}$ film from [9], which showed that they do not satisfy the Wemple-DiDomenico model. On the other hand, more resent computed data for $n(\lambda=210 \div 1250 \mathrm{~nm})$ of films $\left(\mathrm{Gd}_{2} \mathrm{O}_{3}\right)_{\mathrm{x}}:\left(\mathrm{SiO}_{2}\right)_{1-\mathrm{x}}$ for $\mathrm{x}=0 \div 1$ of another rare earth element Gadollinium show that the Wemple-DiDomenico model is satisfied for these films [15]. This controversy would be attributed to drawing inaccurate envelopes of the respective transmittance spectrum in [9].

One difficulty in drawing envelopes of $T(\lambda)$ is that, especially for relatively thin films, the inspected spectrum 
often contains too small number of maxima and minima, advancing the respective computer code, which the which hampers constructing accurate envelopes. To authors of the algorithm [16] received from other thin film address this problem, Minkov and Swanepoel proposed researchers. another algorithm for computer drawing envelopes of $T(\lambda)$, with interference pattern, of a thin film on a transmitting substrate in 1992 [16]. In their algorithm, some 'boundary points' and 'additional points' are also used in constructing both envelopes. 'Boundary points' are calculated at the longest wavelength of the spectrum at $900 \mathrm{~nm}$, assuming that both the film and the substrate are transparent there. In the spectral region of medium absorption in the film, 'additional points' can occur at wavelengths where the first derivative $T^{\prime}(\lambda)>0$ has a local minimum or maximum [16]. In the region of strong absorption in the film, the two envelopes converge, and $T(\lambda) \rightarrow 0$ with decreasing the wavelength. It is recommended only two 'additional points' to be introduced there, but their positions are not specified [16]. The algorithm from [16] uses 'linear' approach and 'parabolic' approach, for creating cubic splines, in the region of negligible film absorption and in the rest of the inspected spectrum, respectively. The algorithm is complicated, employing compound splines to determine a single tangential point, which hinders its applicability.

Notably, the algorithm from [16] was developed for optical characterization employing spectra obtained by UV/visible spectrophotometers. Using UV/visible/NIR spectrophotometers though results in dealing with wider spectra. In particular, increasing the longest wavelength of the spectrum, from $900 \mathrm{~nm}$ to $2500 \mathrm{~nm}$, leads to arising notable absorption in the commonly used glass substrates, especially at $\lambda>1700 \mathrm{~nm}$ [17]. In this case, the boundary conditions for the envelopes at the longest wavelength of the transmittance spectrum, from [16], need some amendment to account for the substrate absorption.

Furthermore, there has been an increasing interest to optical characterization in the region of strong absorption for studying the dielectric properties of films, e.g. for determining the dominant electron transitions through the bandgap by using a Tauc plot [18-19]. This process can be facilitated by drawing more accurate envelopes of $T(\lambda)$ in the region of strong absorption, based on including 'supplementary points' in this region.

The method of Swanepoel was expanded by Marquez et al. in 2002 [17], to account for both the variable thickness of the film, and the absorption in the substrate. The variable film thickness results in shrinking the transmittance spectrum, and the absorption in the substrate leads to decreasing $T_{+}(\lambda)$ and $T_{-}(\lambda)$ by the same factor. Since both of these effects decrease the difference $T_{+}(\lambda)-T_{\text {. }}$ $(\lambda)$ for each $\lambda$, and a new parameter of the variable film thickness is introduced, accurate optical characterization in this general case requires drawing even more accurate envelopes, compared to the original method of Swanepoel [6].

The need for improvement the existing algorithms for computer drawing the envelopes of transmittance spectra is also indicated by the tens of requests, for providing and
The main goal of the study described here was to develop an advanced algorithm for drawing accurate envelopes of transmittance spectrum with interference pattern, of a thin film on a substrate, which addresses all of the described above problems for drawing accurate envelopes. Development of such an algorithm represents a basis for very accurate and model free optical characterization of dielectric, semiconductor, and insulator thin films.

\section{THE ADVANCED ALGORITHM FOR DRAWING ACCURATE ENVELOPES OF TRANSMITTANCE SPECTRA OF THIN FILM ON SUBSTRATE SPECIMENS}

An easy way to comply with the conference paper formatting requirements is to use this document as a template and simply type your text into it.

During the development of such an algorithm, we had three main objectives: selecting a superior interpolation technique for this type of spectra; introducing accurate 'boundary points', 'additional points', and 'supplementary points' allowing drawing accurate envelopes over the entire inspected spectrum; and obtaining a simpler algorithm compared to the algorithms from [9] and [16]. A block diagram of the proposed advanced algorithm, for drawing envelopes of transmittance spectrum with interference pattern of thin film specimens, is shown in Figure 1.

The individual steps of this algorithm are as follows:

2.1 Obtain and digitize the transmittance spectrum $T(\lambda)$. The spectrum is obtained by a spectrophotometer. This process introduces noise to the true spectrum, which is typically within $\pm 0.2 \%$ [20-21].

2.2 Derive smoothed spectrum $T_{\mathrm{sm}}(\lambda)$. This process should eliminate fake maxima and minima, without any notable interference pattern smoothing. Markedly, $T(\lambda)$ of thicker films contains larger number of apparent peaks, due to validity of the interference condition [6]. Moreover, the shoulders of the peaks are wider at longer wavelengths, due to the same reason.

Based on the above, for spectra with more than 10 apparent peaks, we smooth the inspected spectrum by using a 'robust locally weighted scatterplot smoothing approach', i.e. robust LOESS [22]. For spectra with less than 10 apparent peaks, we use 'Savitzky-Golay filter approach' [23] in the region of negligible absorption if the film, where the upper envelope $T_{+}(\lambda)$ does not change significantly with $\lambda$, and LOESS in the rest of the spectrum. For all spectra we dealt with, a suitable choice of the only parameter of the respective smoothing approach leads to completing the smoothing process in just one step of the approach, and no further smoothing is necessary. 


\section{International Advanced Research Journal in Science, Engineering and Technology}

\section{ISO 3297:2007 Certified}

Vol. 3, Issue 9, September 2016

2.3 Find the maxima points $\mathrm{T}_{\mathrm{M}}\left(\lambda_{\mathrm{M}}\right)$ and the minima points $\mathrm{T}_{\mathrm{m}}\left(\lambda_{\mathrm{m}}\right)$ of the smoothed spectrum. All transmittances and wavelengths for the maxima points $T_{M}\left(\lambda_{M}\right)$ of the smoothed spectrum, and for its minima points $T_{m}\left(\lambda_{m}\right)$, are determined by running the freely available 'peakdet' code [24] only once. The only parameter in this code represents the minimal prescribed difference between the transmittances for each apparent adjacent maximum and minimum points. The value of this parameter is chosen by a preliminary inspection of the smoothed spectrum.

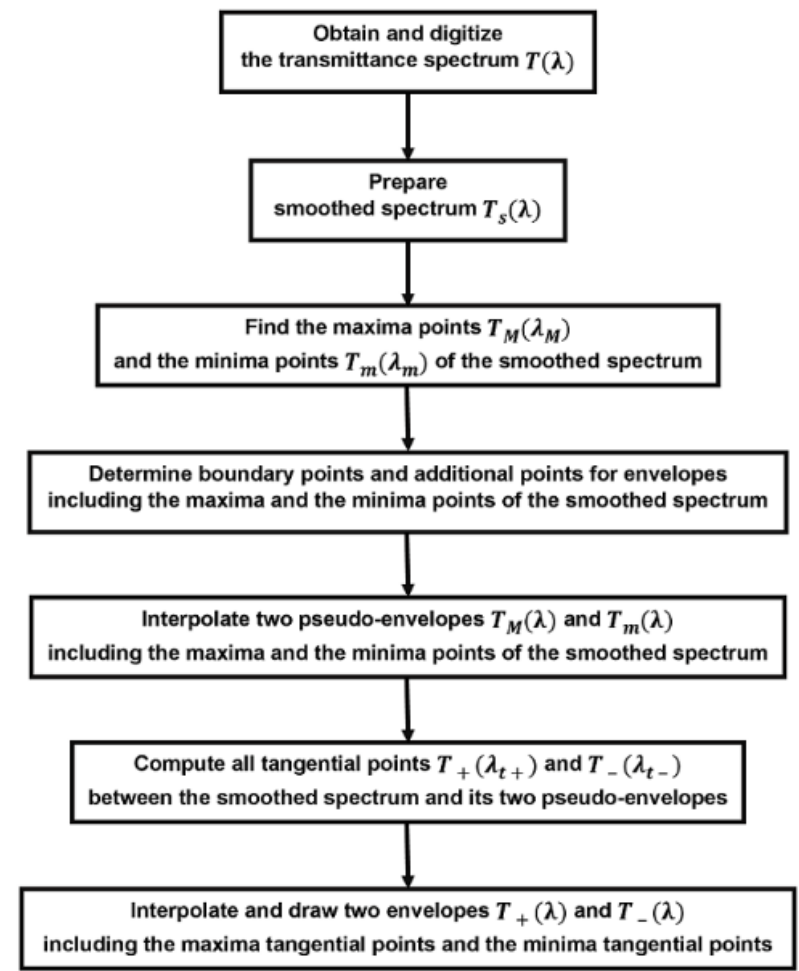

Fig. 1 The advanced algorithm for computer drawing envelopes of transmittance spectrum $\mathrm{T}(\lambda)$ exhibiting interference pattern.

2.4 Determine 'boundary points', 'additional points', and 'supplementary points' for two envelopes of the smoothed spectrum. For negligible absorption in the substrate, in the common case of small variation of the refractive index $n(\lambda)$ in the region of negligible film absorption, the transmittance $\mathrm{T}_{0 \pm}\left(\lambda_{\mathrm{L}}\right)$ at the longest wavelength $\lambda_{\mathrm{L}}$ of the smoothed spectrum, for both its upper envelope and lower envelope, has been expressed as:

$$
\mathrm{T}_{0 \pm}\left(\lambda_{\mathrm{L}}\right)=\mathrm{T}_{0 \pm}\left(\lambda_{1}\right)+\left[\mathrm{T}_{0 \pm}\left(\lambda_{1}\right)-\mathrm{T}_{0 \pm}\left(\lambda_{2}\right)\right] \frac{\left(\lambda_{\mathrm{L}}-\lambda_{1}\right) \lambda_{2}}{\left(\lambda_{1}-\lambda_{2}\right) \lambda_{\mathrm{L}}}
$$

where $\lambda_{1}$ and $\lambda_{2}<\lambda_{1}$ are the wavelengths for the two longest wavelength apparent maxima or minima of the inspected spectrum [16].

When UV/visible/NIR spectrophotometer and glass substrate are used though, the substrate absorption should be taken into account, in the boundary conditions for the envelopes, at $\lambda_{1}$. On the other hand, using flow graphs theory indicates that the following approximation is valid in this case:

$$
\mathrm{T}_{ \pm}\left(\lambda_{\mathrm{i}}\right)=\frac{\mathrm{T}_{0 \pm}\left(\lambda_{\mathrm{i}}\right)}{\mathrm{x}_{\mathrm{S}}\left(\lambda_{\mathrm{i}}\right)}
$$

where $T_{ \pm}$is the transmittance including the substrate absorption for the respective envelope, $\mathrm{x}_{\mathrm{s}}$ is the substrate absorbance, and $\lambda_{\mathrm{i}}=\lambda_{\mathrm{L}}, \lambda_{1}$, or $\lambda_{2}$ [25]. Replacing Eq. (2) in Eq. (1) leads to the following formula for the 'boundary points' $T_{ \pm}\left(\lambda_{L}\right)$ of the two envelopes at the longest wavelength of the smoothed spectrum:

$\mathrm{T}_{ \pm}\left(\lambda_{\mathrm{L}}\right)=\mathrm{x}_{\mathrm{S}}\left(\lambda_{\mathrm{L}}\right)\left\{\frac{\mathrm{T}_{ \pm}\left(\lambda_{1}\right)}{\mathrm{x}_{\mathrm{S}}\left(\lambda_{1}\right)}+\left[\frac{\mathrm{T}_{ \pm}\left(\lambda_{1}\right)}{\mathrm{x}_{\mathrm{S}}\left(\lambda_{1}\right)}-\frac{\mathrm{T}_{ \pm}\left(\lambda_{2}\right)}{\mathrm{x}_{\mathrm{S}}\left(\lambda_{2}\right)}\right] \frac{\left(\lambda_{\mathrm{L}}-\lambda_{1}\right) \lambda_{2}}{\left(\lambda_{1}-\lambda_{2}\right) \lambda_{\mathrm{L}}}\right\}$

Furthermore, Eq. (3) can be rewritten as:

$$
S_{ \pm}\left(\lambda_{L}\right)=\left[\frac{x_{S}\left(\lambda_{L}\right)}{x_{S}\left(\lambda_{1}\right)}-1\right] \frac{T_{ \pm}\left(\lambda_{1}\right)}{\left(\lambda_{L}-\lambda_{1}\right)}+\left[\frac{T_{ \pm}\left(\lambda_{1}\right)}{x_{S}\left(\lambda_{1}\right)}-\frac{T_{ \pm}\left(\lambda_{2}\right)}{x_{S}\left(\lambda_{2}\right)}\right] \frac{\lambda_{2} x_{S}\left(\lambda_{L}\right)}{\left(\lambda_{1}-\lambda_{2}\right) \lambda_{L}}
$$

where $S_{ \pm}\left(\lambda_{L}\right)$ is the boundary slope of the respective envelope at $\lambda_{\mathrm{L}}$. Therefore, Eq. (3) and Eq. (4) represent the boundary condition at the longest wavelength of the smoothed spectrum, provided that the substrate absorbance $\mathrm{x}_{\mathrm{s}}(\lambda)=\exp \left(-4 \pi \mathrm{k}_{\mathrm{s}} \mathrm{t}_{\mathrm{s}} / \lambda\right)$ is known, where $\mathrm{k}_{\mathrm{s}}(\lambda)$ is the extinction coefficient of the substrate, and $t_{s}$ is its thickness.

In the region of medium film absorption, for wavelengths smaller than all apparent maxima and minima of the inspected spectrum i.e. $\lambda<\min \left(\lambda_{\mathrm{M}}, \lambda_{\mathrm{m}}\right)$, both the transmittance and the distance between the two envelopes decrease with decreasing $\lambda$, due to increasing film absorption. Therefore, there can often be included one or even two pairs of 'additional points' $\mathrm{T}_{\mathrm{M}}\left(\lambda_{\mathrm{Mad}}\right)$ and $\mathrm{T}_{\mathrm{m}}\left(\lambda_{\mathrm{mad}}\right)$ for constructing more accurate upper and lower envelope, respectively. $\mathrm{T}_{\mathrm{M}}\left(\lambda_{\mathrm{Mad}}\right)$ and $\mathrm{T}_{\mathrm{m}}\left(\lambda_{\mathrm{mad}}\right)$ correspond to a maximum and a minimum of the first derivative of the smoothed spectrum, which has a positive value $\mathrm{T}_{\mathrm{sm}}{ }^{\prime}(\lambda)>0$ in this spectral region. These 'additional points' were introduced in [16], and are determined here by using the 'peakdet' code with respect to the first derivative of the smoothed spectrum. With further decreasing $\lambda$ into the region of strong film absorption, both $\mathrm{T}_{\mathrm{sm}}(\lambda)$ and its first derivative $\mathrm{T}_{\mathrm{sm}}{ }^{\prime}(\lambda)$ decrease towards zero, and the two envelopes converge. It turns out that the 'convergence point' $\mathrm{T}_{ \pm}\left(\lambda_{\mathrm{C}}\right)$ can be defined as:

$$
\mathrm{T}_{ \pm}{ }^{\prime}\left(\lambda_{\mathrm{C}}\right)=\max \left[\mathrm{T}_{\mathrm{sm}}{ }^{\prime}(\lambda)\right], \text { for } \lambda<\min \left(\lambda_{\mathrm{M}}, \lambda_{\mathrm{Ma}}\right)
$$

or as the wavelength $\lambda_{\mathrm{C}}$ providing a maximal second derivative of the smoothed spectrum in the region of strong film absorption. Moreover, all points $\mathrm{T}_{ \pm}\left(\lambda_{\mathrm{Ss}}\right)$ from the smoothed spectrum for which:

$0<\mathrm{T}_{ \pm}\left(\lambda_{\mathrm{Ss}}\right) \leq \mathrm{T}_{ \pm}\left(\lambda_{\mathrm{C}}\right)$, or $\lambda_{\mathrm{S}} \leq \lambda_{\mathrm{Ss}} \leq \lambda_{\mathrm{C}}$

where $\lambda_{S}$ is the shortest wavelength of the inspected spectrum, i.e. $\mathrm{T}_{\mathrm{sm}}\left(\lambda_{\mathrm{S}}\right)>0$, should be used as 'supplementary points' for drawing accurate envelopes in the region of strong film absorption. Therefore, all 'supplementary points' $\mathrm{T}_{ \pm}\left(\lambda_{\mathrm{Ss}}\right)$ and their respective slope $\mathrm{S}_{ \pm}\left(\lambda_{\mathrm{Ss}}\right)$ of the smoothed spectrum represent the boundary condition for both envelopes at the short wavelength end of the inspected spectrum. 


\section{International Advanced Research Journal in Science, Engineering and Technology} ISO 3297:2007 Certified

Vol. 3, Issue 9, September 2016

2.5 Interpolate two pseudo-envelopes $\mathrm{T}_{\mathrm{M}}(\lambda)$ and $\mathrm{T}_{\mathrm{m}}(\lambda)$ along the maxima and the minima points of the smoothed spectrum. At the beginning of this step, all maxima points $\mathrm{T}_{\mathrm{M}}\left(\lambda_{\mathrm{M}}\right)$, 'boundary point', 'additional points', and 'supplementary points' for the upper envelope are set in order of decreasing their wavelengths. A pseudo-envelope $T_{M}(\lambda)$ is interpolated next to pass through all of these points, spreading over the entire inspected spectrum, by using a 'piecewise cubic Hermite interpolating polynomial' (PCHIP) [25]. The same procedure is repeated then for interpolating a pseudo-envelope $T_{m}(\lambda)$ passing through all minima points $\mathrm{T}_{\mathrm{m}}\left(\lambda_{\mathrm{m}}\right)$, 'boundary point', 'additional points', and 'supplementary points' for the lower envelope.

In most practical cases, the film absorption increases sharply with decreasing the wavelength, which leads to sharply decreasing transmittance [6]. On the other hand, PCHIP is perceived to interpolate better a sharply changing function, compared to 'piecewise cubic spline' (PCS) [26]. Due to the above two reasons, we employ mostly PCHIP interpolation in this paper. It should be noted, that this PCHIP does not use the slopes $S_{ \pm}\left(\lambda_{L}\right)$ and $S_{ \pm}\left(\lambda_{S s}\right)$ of the smoothed spectrum, at both ends of the inspected spectrum, but PCS interpolation uses them.

2.6 Compute the tangential points $\mathrm{T}_{+}\left(\lambda_{\mathrm{t}}\right)$ and $\mathrm{T}_{-}\left(\lambda_{\mathrm{t}}\right)$ between the smoothed spectrum and its two pseudoenvelopes. In the beginning of this step are calculated the first derivatives of the smoothed spectrum and its two pseudo-envelopes, for all wavelengths of the inspected spectrum. The function $\mathrm{D}_{\mathrm{M}}(\lambda)=\left|\mathrm{T}_{\mathrm{sm}}{ }^{\prime}(\lambda)-\mathrm{T}_{\mathrm{M}}{ }^{\prime}(\lambda)\right|$ represents an absolute value of the difference between the first derivatives of the transmittance of the smoothed spectrum, and of its pseudo-envelope along the maxima points. The tangential points $T_{+}\left(\lambda_{t+}\right)$ between $T_{s m}(\lambda)$ and $\mathrm{T}_{\mathrm{M}}(\lambda)$ are computed next by finding the local minima of the function $D_{M}$ with respect to the wavelength, whereat $\lambda_{\mathrm{C}}<\lambda_{\mathrm{t}}<\lambda_{\mathrm{L}}$, using the 'peakdet' code. The only parameter in this code represents the minimal prescribed value of this function around each of the apparent maxima of $\mathrm{T}_{\mathrm{sm}}(\lambda)$. The value of this parameter is chosen by a preliminary inspection of the function $D_{M}(\lambda)$. The same procedure is repeated then for computing the tangential points $T_{-}\left(\lambda_{t-}\right)$ between $T_{s m}(\lambda)$ and $T_{m}(\lambda)$, by finding the local minima of the function $\mathrm{D}_{\mathrm{m}}(\lambda)=\left|\mathrm{T}_{\mathrm{sm}}(\lambda)-\mathrm{T}_{\mathrm{m}}(\lambda)\right|$ with respect to the wavelength, whereat $\lambda_{\mathrm{C}}<\lambda_{\mathrm{t}-}<\lambda_{\mathrm{L}}$. As a part of computing the tangential points between the smoothed spectrum and its two pseudo-envelopes, the possible pairs of additional points $\mathrm{T}_{\mathrm{M}}\left(\lambda_{\mathrm{Mad}}\right)$ and $\mathrm{T}_{\mathrm{m}}\left(\lambda_{\mathrm{mad}}\right)$ become $\mathrm{T}_{+}\left(\lambda_{\mathrm{Ma}}\right)$ and $\mathrm{T}_{-}\left(\lambda_{\mathrm{ma}}\right)$, respectively.

2.7 Interpolate and draw two envelopes $T_{+}(\lambda)$ and $T_{-}(\lambda)$ along the maxima tangential points and the minima tangential points. The tangential points $T_{+}\left(\lambda_{t+}\right)$, the 'boundary point' $\mathrm{T}_{+}\left(\lambda_{\mathrm{L}}\right)$, and the 'supplementary points' $\mathrm{T}_{+}\left(\lambda_{\mathrm{Ss}}\right)$ are considered to belong to the upper envelope of the spectrum $T(\lambda)$. Therefore, the upper envelope $T_{+}(\lambda)$ is interpolated to pass through all of these points, spreading over the entire inspected spectrum, by using PCHIP.
Similarly, the lower envelope $T_{-}(\lambda)$ of the spectrum is interpolated, by using PCHIP, to pass through all of the tangential points $\mathrm{T}_{-}\left(\lambda_{\mathrm{t}}\right)$, the 'boundary point' $\mathrm{T}_{-}\left(\lambda_{\mathrm{L}}\right)$, and the 'supplementary points' $\mathrm{T}_{-}\left(\lambda_{\mathrm{Ss}}\right)$.

In the next two chapters are shown four Figures containing a transmittance spectrum, and its computer drawn envelopes. On these Figures are represented by open circles: all of the tangential points between the respective smoothed spectrum and its two envelopes; the 'boundary points' $T_{+}\left(\lambda_{L}\right)$ and $T_{-}\left(\lambda_{L}\right)$; the possible pairs of 'additional points' $T_{+}\left(\lambda_{\mathrm{Ma}}\right)$ and $\mathrm{T}_{-}\left(\lambda_{\mathrm{ma}}\right)$; and the 'convergence point' $\mathrm{T}_{ \pm}\left(\lambda_{\mathrm{C}}\right)$.

\section{DRAWING ENVELOPES FOR MODEL SPECIMENS}

The performance of the proposed algorithm is demonstrated for two model specimens representing one thin semiconductor film, with refractive index $\mathrm{n}(\lambda)=\frac{3 \times 10^{5}}{\lambda^{2}}+2.6$ and extinction coefficient $\mathrm{k}(\lambda)=$ $\frac{\lambda}{4 \pi} \times 10^{\left(1.5 \times 10^{6} / \lambda^{2}-8\right)}$, positioned on a transparent substrate, with refractive index $\mathrm{n}_{\mathrm{s}}(\lambda)=1.51$ and extinction coefficient $\mathrm{k}_{\mathrm{s}}(\lambda)=0$, where $\lambda(\mathrm{nm})$, at normal incidence of spectrophotometric light [16]. The extinction coefficient of this film increases sharply with decreasing the wavelength, which leads to sharply declining envelopes in the short wavelength side of the transmittance spectrum [6], thus imposing challenges to computer drawing its envelopes.

The transmittance spectrum of such model specimen, and its two known true envelopes are calculated, for a given thickness of the film, using formulae presented in [27]. Moreover, $\pm 0.2 \%$ noise is added to this calculated transmittance spectrum, to emulate the spectrophotometric noise. Since the number of apparent peaks in the spectrum $T(\lambda)$ is proportional to the thickness of the film, due to the interference condition, drawing accurate envelopes of transmittance spectrum is more difficult for thinner films. Therefore, initially is investigated drawing envelopes, for the first model specimen, when the thickness of the film is $\mathrm{d}=500 \mathrm{~nm}$. In this case, the following types of upper and lower envelopes of the transmittance spectrum are computed: the known true envelopes of $\mathrm{T}(\lambda)$, envelopes obtained using PCHIP interpolation at steps 2.5 and 2.7 of the algorithm, and envelopes using PCS interpolation at steps 2.5 and 2.7. It should be noted that the used PCHIP interpolation does not employ any boundary slopes of the smoothed spectrum, whereas the used PCS employs $S_{ \pm}\left(\lambda_{L}\right)$ and $S_{ \pm}\left(\lambda_{S}\right)$. The noisy transmittance spectrum, and its respectively computed envelopes are shown in Figure 2. In this case, one pair of 'additional points' $\mathrm{T}_{\mathrm{M}}\left(\lambda_{\mathrm{Ma}}\right)$ and $\mathrm{T}_{\mathrm{m}}\left(\lambda_{\mathrm{ma}}\right)$ is included, before interpolating the respective pseudo-envelopes $\mathrm{T}_{\mathrm{M}}(\lambda)$ and $\mathrm{T}_{\mathrm{m}}(\lambda)$, when using either of PCHIP or PCS interpolation

As seen from Figure 2, using PCHIP interpolation provides accurate envelopes, positioned close to the respective true envelopes, even for a relatively thin film with sharply declining envelopes in the short wavelength side of the transmittance spectrum. 


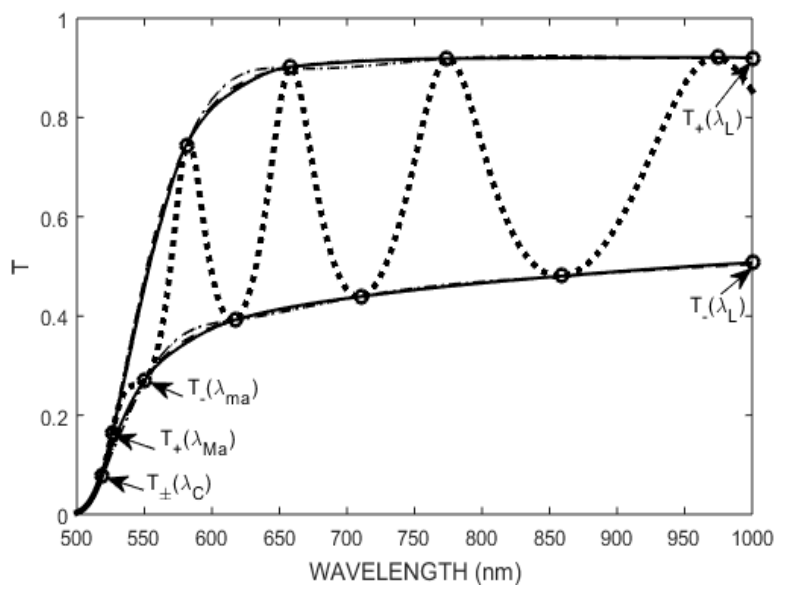

Fig. 2 Transmittance spectrum for the first model specimen with a film thickness $\mathrm{d}=500 \mathrm{~nm}$ and $\pm 0.2 \%$ noise, and its envelopes. $\mathrm{T}(\lambda)$ - dotted line (....); the true envelopes of $\mathrm{T}(\lambda)$ - dashed lines (- - -); the envelopes obtained using PCHP interpolation at steps 1.5 and 1.7 of the algorithm - solid lines (__ $)$; and the envelopes obtained using PCS interpolation at steps 1.5 and 1.7 dash-dot lines (---.-).

Apparently, using PCS interpolation leads to drawing inaccurate envelopes in the short wavelength side of the reflection spectrum. Moreover, the data from Figure 2 indicate that using PCHIP provides accurate envelopes, in comparison with using PCS, even though PCS employs boundary slopes of the smoothed spectrum, and PCHIP does not employ boundary slopes. Another noisy transmittance spectrum, its known true envelopes, and PCHIP interpolated envelopes are represented in Figure 3, for the second model specimen with a film thickness $\mathrm{d}=$ $1000 \mathrm{~nm}$. In this case, there are no 'additional points' $\mathrm{T}_{\mathrm{M}}\left(\lambda_{\mathrm{Ma}}\right)$ and $\mathrm{T}_{\mathrm{m}}\left(\lambda_{\mathrm{ma}}\right)$ included, before interpolating the respective pseudo-envelopes $\mathrm{T}_{M}(\lambda)$ and $\mathrm{T}_{\mathrm{m}}(\lambda)$.

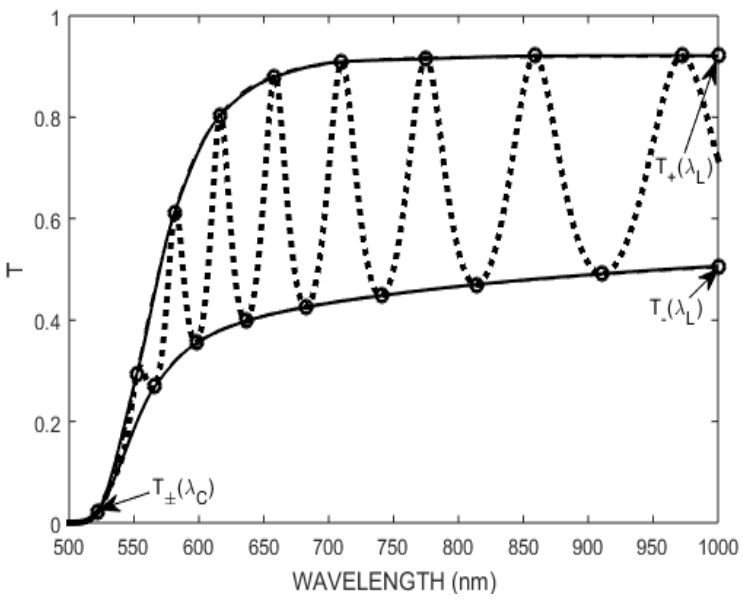

Fig. 3 Transmittance spectrum for the second model specimen with a film thickness $d=1000 \mathrm{~nm}$ and $\pm 0.2 \%$ noise, and its envelopes. $\mathrm{T}(\lambda)$ - dotted line (....); the true envelopes - dashed lines (- - -); and the PCHIP interpolated envelopes - solid lines (_ $\left({ }_{\text {) }}\right.$.
As seen from Figure 3, PCHIP interpolation provides almost perfect envelopes for the second model specimen with $1000 \mathrm{~nm}$ thick film. Moreover, a comparison between Figure 2 and Figure 3 indicates that PCHIP interpolated envelopes are more accurate for thicker films.

\section{DRAWING ENVELOPES OF EXPERIMENTAL TRANSMITTANCE SPECTRA}

To exhibit the performance of the algorithm for drawing envelopes to actual specimens, envelopes are PCHIP interpolated and drawn for two experimental transmittance spectra. A transmittance spectrum, and its two envelopes is shown in Figure 4a, for a vacuum sputtered a-Si film with a thickness of about $780 \mathrm{~nm}$ on $0.9 \mathrm{~mm}$ thick CNG09 Corning glass substrate. In this case, one pair of 'additional points' $\mathrm{T}_{\mathrm{M}}\left(\lambda_{\mathrm{Ma}}\right)$ and $\mathrm{T}_{\mathrm{m}}\left(\lambda_{\mathrm{ma}}\right)$ is included, before interpolating the respective pseudo-envelopes $\mathrm{T}_{M}(\lambda)$ and $T_{m}(\lambda)$.

Another transmittance spectrum, and its two envelopes is represented in Figure 4b, for another vacuum sputtered aSi film with a thickness of about $3930 \mathrm{~nm}$ on $3.28 \mathrm{~mm}$ thick Borofloat33 Schott glass substrate. In this case, there are no 'additional points' $\mathrm{T}_{\mathrm{M}}\left(\lambda_{\mathrm{Ma}}\right)$ and $\mathrm{T}_{\mathrm{m}}\left(\lambda_{\mathrm{ma}}\right)$ included, before interpolating the pseudo-envelopes $T_{M}(\lambda)$, and $T_{m}(\lambda)$.

Figure 4 illustrates that the algorithm for computer drawing envelopes of transmittance spectra, with interference pattern, provides envelopes with a good quality, for thin films with disparate thicknesses.

(a)

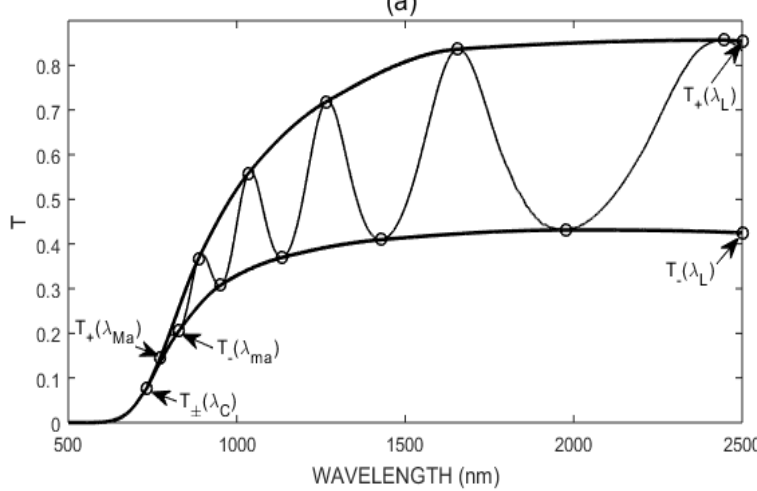

(b)

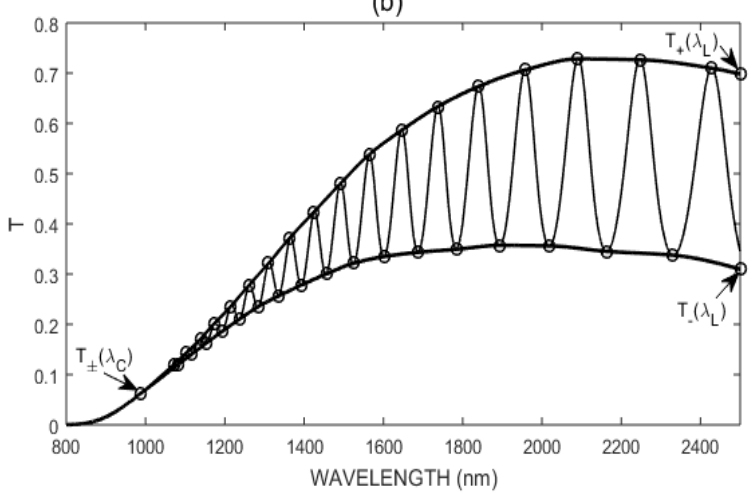


Vol. 3, Issue 9, September 2016

Fig. 4 Transmittance spectrum and its two PCHIP interpolated envelopes, for: (a) about $780 \mathrm{~nm}$ thick a-Si film on $0.9 \mathrm{~mm}$ thik CNG09 Corning glass substrate. (b) about $3930 \mathrm{~nm}$ thick a-Si film on $3.28 \mathrm{~mm}$ thick Borofloat33 Schott glass substrate.

\section{DISCUSSION}

The proposed algorithm for computer drawing envelopes of transmittance spectra $T(\lambda)$ with interference pattern, of specimens representing a thin film on a substrate, has several advantages with respect to the existing algorithms [9] and [16], as a result of its following features:

- It is shown here that PHIP interpolation provides envelopes which fit better the true envelopes of such spectra, than the conventional PCS interpolation. This is due to the disparate values of the first derivative $T^{\prime}(\lambda)$ in the regions of small, medium, and strong absorption in the film. Therefore, PCHIP interpolation is employed in this algorithm.

- It uses accurate 'boundary points' $T_{ \pm}\left(\lambda_{L}\right)$ at the longest wavelength of the spectrum. This is achieved by taking into account the substrate absorption, since it is usually larger in the near-infrared and the short-infrared regions, than in the UV and the visible regions. Besides, using accurate 'boundary points' at $\lambda_{\mathrm{L}}$ leads to accurate determination of the tangential points around the first apparent maximum and minimum, adjacent to $\lambda_{\mathrm{L}}$, which might not be the case e.g. when no boundary conditions are used.

- The 'convergence point' $T_{ \pm}\left(\lambda_{C}\right)$ is introduced, where the two envelopes converge, in the region of strong film absorption. The use of $T_{ \pm}\left(\lambda_{C}\right)$, whereas including the possible pairs of 'additional points' $\mathrm{T}_{\mathrm{M}}\left(\lambda_{\mathrm{Ma}}\right)$ and $\mathrm{T}_{\mathrm{m}}\left(\lambda_{\mathrm{ma}}\right)$, increases the envelopes accuracy in the medium film absorption region.

- All points of the smoothed spectrum, for wavelengths $\lambda_{\mathrm{S}} \leq \lambda_{\mathrm{Ss}} \leq \lambda_{\mathrm{C}}$, are introduced and used as 'supplementary points' $\mathrm{T}_{ \pm}\left(\lambda_{\mathrm{Ss}}\right)$. This preserves the whole transmittance spectrum information in the strong film absorption region.

- It is simpler mathematically, since it smooths the spectrum in only one step, does not use multi-step iteration, neither compound spline.

\section{CONCLUSION}

An advanced algorithm is proposed for computer drawing envelopes of transmittance spectra, with interference pattern, of specimens of a thin film on a transmitting substrate. It allows attaining accurate envelopes over the entire spectrum, unlike the existing algorithms [9] and [16]. The development of this algorithm represents a basis for very accurate and model free optical characterization of thin dielectric, semiconductor, and insulator thin films. Our unpublished results show that using this algorithm leads to optical characterization of films with significant thickness variation and notable substrate absorption, which has been precarious using the existing algorithms for computer drawing envelopes of transmittance spectra [17].

\section{ACKNOWLEDGMENT}

The two a-Si film specimens have been prepared at CIEMAT in Madrid, Spain.

\section{REFERENCES}

[1] J. Tominaga and T. Nakano, Optical Near-Field Recording (Springer, Heidelberg, 2005).

[2] R. G. Hunsperger, Integrated Optics: Theory and Technology, 6th ed. (Springer-Verlag, New York, 2009).

[3] M. Wakaki, Optical Materials and Applications (CRC Press, Boca Raton, 2012).

[4] H. A. McLeod, Thin-Film Optical Filters, 4th ed. (CRC Press, London, 2010).

[5] D. Poelman and P. J. Smet, J. Phys. D. 36, 1850-1857 (2003).

[6] R. Swanepoel, J. Phys. E. 16, 1214-1222 (1983).

[7] L. M. Manceriu, A. Rougier, and A. Duta, J. of Alloys Compounds 630, 133-145 (2015).

[8] D. Minkov, Optik 88, 60-62 (1991).

[9] M. McClain, A. Feldman, D. Kahaner, and X. Ying, Computers in Physics 5, 45-48 (1991).

[10] T. Güngör, and B. Saka, Thin Solid Films 467, 319-325 (2004).

[11] A. M. El-Naggar, S. Y. El-Zaiat, and S. M. Hassan, Optics and Laser Technology 41, 334-338 (2009).

[12] S. H. Wemple and M. DiDomenico, Jr., Phys. Rev B 3, 1338-1351 (1971).

[13] G. E. Jellison Jr. and F. A. Modine, Appl. Phys. Lett. 69, 371-373 (1996).

[14] P. Petkova and K. Boubaker, J. Alloys and Compounds 546 176179 (2013).

[15] N. K. Sahoo, S. Thakur, R. B. Tokas, A. Biswas, and N. M. Kamble, Appl. Surface Science 253, 3455-3463 (2007).

[16] D. Minkov and R. Swanepoel, Proc. SPIE 1782, 212-220 (1993).

[17] J. M. G. Leal, R. P. Alcon, J. A. Angel, D. A. Minkov, and E. Marquez, Appl. Optics 41, 7300-7308 (2002).

[18] N. Song, Y. Wang, Y. Hu, et al., Appl. Phys. Lett. 104, 092103 (2014).

[19] M. I Saidaminov, A. L. Abdelhady, B. Murali, et al., Nature Communications 6, 7586 (2015).

[20] Demonstrating the accuracy of transmittance measurements for high refractive index materials: Technical Note (Perkin-Elmer Inc., Waltham, 2010).

[21] Instrument Manual for the Lambda 19 Perkin-Elmer UV/Vis/NIR Spectrometer (Perkin-Elmer GmbH, Waltham, 1990).

[22] W. S. Cleveland, J. American Statistical Association 74, 829-836 (1979).

[23] H. H. Madden, Analytical Chemistry 50, 1383-1386 (1978).

[24] E. Billauer, Toolbox for analysis of spectroscopy data, ed. 3.4.05. http://www.mathworks.com/matlabcentral/fileexchange/32828spectr-o-matic/content/peakdet.m (MATLAB Central, 2015).

[25] D. Minkov, Appl. Optics 33, 7698-7703 (1994).

[26] F. N. Fritsch and R. E. Carlson, SIAM J. Numerical Analysis 17, 238-246 (1980).

[27] S. Moler, Splines and Pchips. http://blogs.mathworks.com/cleve/ 2012/07/16/splines-and-pchips/ (MATLAB Central, 2012). 